\title{
Study on Measurement of Group Delay in Broadband Channel Based on Nonlinear Fitting
}

\author{
Xinfeng Mao ${ }^{a}$, Hong $\mathrm{Ma}^{\mathrm{b}}{ }^{\text {, Ge Song }}{ }^{\mathrm{c}}$ and Baotong Peng ${ }^{\mathrm{d}}$ \\ School of Equipment Academy, Beijing 101416, China \\ amaomaohao60@163.com, bmahong_0108@163.com, \\ clovesg9251@163.com, d13810479417@163.com
}

\begin{abstract}
In VLBI, obtaining group delay characteristics of equipment is essential to ensure the measurement accuracy. The thesis proposes a new measurement method, nonlinear fitting method, by analyzing how to get group delay characteristics with direct phase difference method and linear-least-squares fitting method. The result of simulation shows that the method measure group delay two orders of magnitude is more accurate than difference method. Actual data demonstrates that difference method and linear fitting method lose effectiveness in frequency from $270 \mathrm{MHz}$ to $370 \mathrm{MHz}$. Otherwise, the nonlinear fitting method's measurement precision of group delay is better than $0.1 \mathrm{~ns}$.
\end{abstract}

Keywords: Group delay; Fitting; Broadband channel; PCAL.

\section{Introduction}

VLBI (Very Long Baseline Interferometry), an important interferometry measurement technology applied to deep space exploration, calculates the precise angle by measuring geometric delay of radiostar among multiple distributed geographically observation stations on earth [1]. Central to VLBI is to process cross correlative data from different observation stations for cross correlative spectrum and delay inequality, that is, geometric delay estimate [2]. The estimate includes not only the true value of geometric delay, but also delay inequality of multiple distributed geographically observation stations' equipment receiving chain. Firstly, the inconsistencies of group delay in different stations receiving chain results that the delay inequality in geometric delay's estimate between equipment receiving chains cannot be eliminated, and affecting the estimation accuracy of geometric delay. The other, the deviations of conventional methods in measuring the group delay of equipment chain are much larger, thereby affecting the estimate of delay inequality between equipment receiving chains, and affecting the accuracy of geometric delay's estimate. Now VLBI mainly adopts direct phase difference method [3] (called difference method below) and linear-least-squares fitting method [4]. Difference method only accesses to phases of two frequency points with low precision and conflicts between precision and resolution are inevitable. Although linear-least-squares fitting method can make up the shortcomings of difference method, it is only applicable for linear phase system and unable to measure characteristics of frequency distribution in group delay of nonlinear phase system.

In order to compensate for the two deficiencies, the thesis proposes a measurement based on nonlinear fitting group delay. The features extraction from addictive phase and group delay in receiving channels of some Station will be obtained with PCAL [5] (Phase Calibration) signal and the nonlinear fitting method to fit the unwrapped phase obtained from PCAL [6], finally, get the characteristic from addictive phase and group delay. The method is not only applicable for nonlinear phase system, but also improves accuracy of group delay making full use of all frequency points of phases.

\section{Research on extraction from group delay}

\subsection{Difference method and linear fitting measurement.}

Difference method, based on definition of group delay, estimates group delay with phase difference of two frequency points and ratio of frequency difference. There is an equation below: 


$$
\left\{\begin{array}{l}
\theta=\Phi(\omega) \\
\tau(\omega)=-\frac{\Delta \theta}{\Delta \omega}=-\frac{\Phi(\omega+\Delta \omega / 2)-\Phi(\omega-\Delta \omega / 2)}{\Delta \omega}
\end{array}\right.
$$

$\Phi(\omega)$ represents phase estimation of angular frequency $\omega$, and $\tau(\omega)$ stands for group delay estimation of angular frequency interval $\Delta \omega$. According to above equation, it can draw an estimated accuracy as follows (an hypothesis that frequency phase estimated variance is $\sigma_{\varphi}^{2}$ ):

$$
\left\{\begin{array}{l}
\sigma_{\theta}^{2}=\sigma_{\varphi}^{2} \\
\sigma_{\tau}^{2}=\frac{2 \sigma_{\varphi}^{2}}{\Delta \omega^{2}}
\end{array}\right.
$$

To distinguish two signals in practical application, the closer the two frequency point signals are, the higher resolution of the device is. With a certain resolution, smaller $\Delta \omega$ is, the smaller Signal-to-Noise Ratio (SNR) of relevant PCAL [7] is $\sigma_{\varphi}^{2}$, the bigger is, which causes inaccuracy of group delay. $\Delta \omega$ cannot be too big to manifest characteristics embodied by frequency distributions. In conclusion, it is difficult to increase the accuracy of group delay with the method due to the above problems.

The critical reason for low accuracy is that the method only accesses phase information of two frequency points rather than makes full use of all phase information according to its definition. Fitting demonstrates phase-frequency curve with phase information of multiple frequency points. The body of linear fitting is a curve based on rules of least squares and $3 \sigma$. VLBI will get cross correlative spectrum of two antennas with cross correlative processing. Slope and offset are respectively corresponding to receiving group delay and measurement accuracy of receiving signals [8]. Even if linear fitting method compensates for shortcomings of difference method, while for VLBI, it is useless for broadband system whose wide-band channels belong to nonlinear phase systems.

From the above, while estimating group delay, it is uneasy to guarantee accuracy for the conflict of difference method between resolution and accuracy, and inapplicable for wide-band channel (non-linear phase system) with linear fitting method. Therefore, the thesis puts forward a measurement based on nonlinear fitting method to correspond to VLBI wide-band channel.

\subsection{Nonlinear fitting measurement.}

According to equation (1), group delay can be characterized as a differential of phase-frequency curve about frequency. It is beneficial to get precise figure with precise phase-frequency curve. The equation is as follows:

$\Phi(\omega)=a_{0}+a_{1}\left(\omega-\omega_{c}\right)+a_{2}\left(\omega-\omega_{c}\right)^{2}+a_{3}\left(\omega-\omega_{c}\right)^{3}+\cdots$

In equation (3), $a_{i}$ is sub phase term, and $\omega_{c}$ is central angle frequency. We can conclude $\tau(\omega)$ as follows:

$\tau(\omega)=-\Phi^{\prime}(\omega)=-a_{1}-2 a_{2}\left(\omega-\omega_{c}\right)-3 a_{3}\left(\omega-\omega_{c}\right)^{2}+\cdots$

The constant term $a_{1}$ in equation (4) indicates the same shifted phase to all frequency points in the system. The shifted phase does not result in non-linear changes delay difference between two different stations. In contrast, the other terms change by time and result in non-linear changes delay.

To sum up, influences of $a_{i}$ should be taken into consideration in phase system. Particularly, $a_{i}(i \geq 2)$ results in big nonlinear delay difference deviation. Consequently, $\Phi^{\prime}(\omega)$ should be fitted to estimate $\vec{a}_{i}$. With power series expansion principle [9], the equation is as follows:

$$
a_{0}=\left.\Phi(\omega)\right|_{\omega=\omega_{c}}, a_{1}=\left.\frac{d \Phi(\omega)}{d \omega}\right|_{\omega=\omega_{c}}, a_{2}=\frac{1}{2 !} \times\left.\frac{d \Phi^{2}(\omega)}{d \omega^{2}}\right|_{\omega=\omega_{c}}, a_{3}=\frac{1}{3 !} \times\left.\frac{d \Phi^{3}(\omega)}{d \omega^{3}}\right|_{\omega=\omega_{c}}, \cdots
$$

As shown in equation (5), group delay estimate $\bar{\tau}(\omega)$ can be obtained by $\hat{a}_{i}$.

\section{Simulation study and performance comparison}

\subsection{Simulation conditions.}

For normality, simulating channels are based on plural signals, sampling frequency being $640 \mathrm{MHz}$, range of measurement frequency being $\pm 250 \mathrm{M}$. Channels supposed to be tested access to group delay 
all-pass filter with 256 order and the maximum group delay of band edge is 20ns. Assuming that coefficient of group delay is the second order and characteristic curve of group delay takes the form of parabola style. The formula of group delay in channels under test is as following [10]:

$$
\begin{aligned}
\tau(f) & =\frac{20 \mathrm{~ns}}{(320 \mathrm{MHz})^{2}} \times f^{2}+\left(\frac{256}{640 \mathrm{MHz}}-\frac{20 \mathrm{~ns}}{3}\right) \\
& \approx \frac{20 \mathrm{~ns}}{(320 \mathrm{MHz})^{2}} \times f^{2}+393.33 \mathrm{~ns}
\end{aligned}
$$

It can be concluded that the range of channel's group delay varies from $393.33 \mathrm{~ns}$ to $413.33 \mathrm{~ns}$ with the frequency varying $\pm 320 \mathrm{MHz}$.

The structure figure below shows channels under test how to add simulating signals:

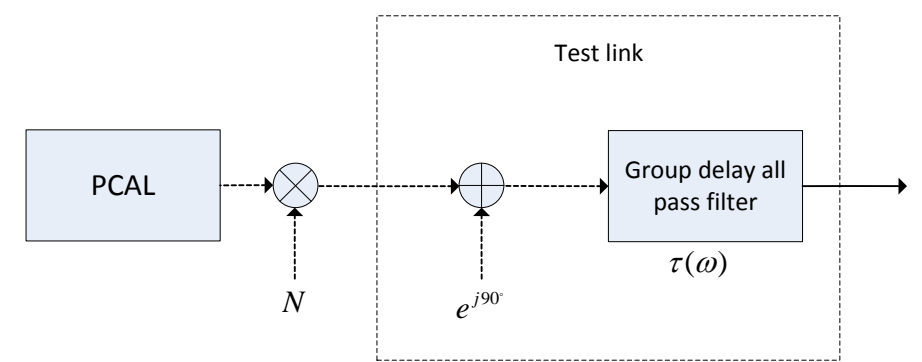

Fig. 1 Simulating figure for characteristic measurement of group delay

As the Fig. 2 shows, group delay $\tau(f)$ of channels under test will be obtained after PCAL signals (frequency interval $\Delta \omega$ being $1 \mathrm{MHz}$ which guarantees that the biggest unambiguous group delay[11] measured is 1us) and White Gaussian Noise $N$ (Signal to Noise Ratio being -30dB, range of frequency being $\pm 300 \mathrm{MHz}$ ) being injected into channels under test simultaneously, addictive phase $\theta=90^{\circ}$ added, which will be generated by all-pass filters.

\subsection{Performance Analysis.}

Phase-frequency curve can be drawn by extracting phases from PCAL signals of output under the guidance of phase extracting technique [6]. When phases are extracted, parameters are respectively: DFT spectral resolution $f_{B W}=1 \mathrm{MHz}$, accumulating times being 2000, and count of phase-frequency curve extracting being 640. There is a figure of phase-frequency curve before and after being unwrapped as follows:
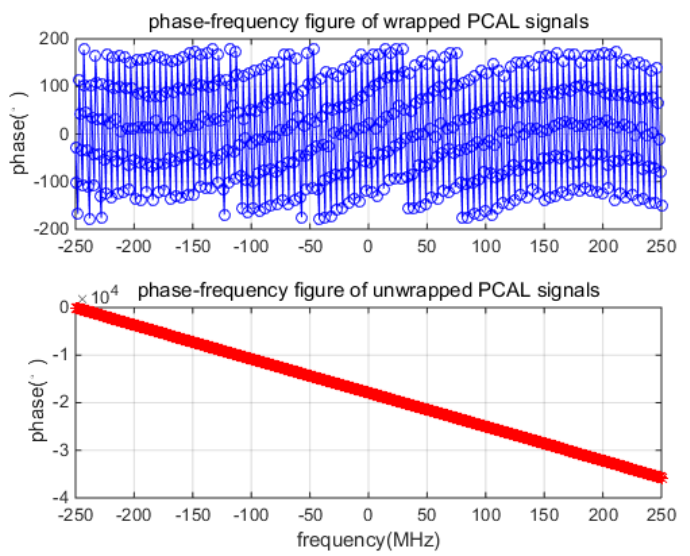

Fig. 2 Phase-frequency figure for wrapped and unwrapped PCAL signal

As the Fig. 2 shows, based on PCAL signal's phase-frequency curve unwrapped, characteristic curve of group delay can be calculated with difference method, linear fitting method and non-linear fitting method respectively.

1000 Monte Carlo simulation experiments were carried out with linear fitting method and nonlinear fitting method respectively, which concluded characteristics of phase fitting residual and residual's standard deviation in wide-band system. The Fig. 3 shows fitting residual characteristics of linear fitting method and nonlinear fitting method and the Fig. 4 shows standard deviation of 1000 Monte Carlo simulation with two methods. 


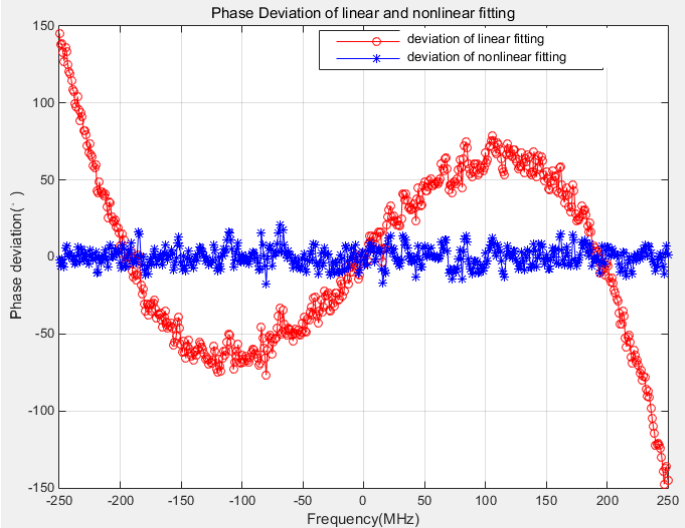

Fig. 3 Comparing figure for linear and nonlinear fitting deviations

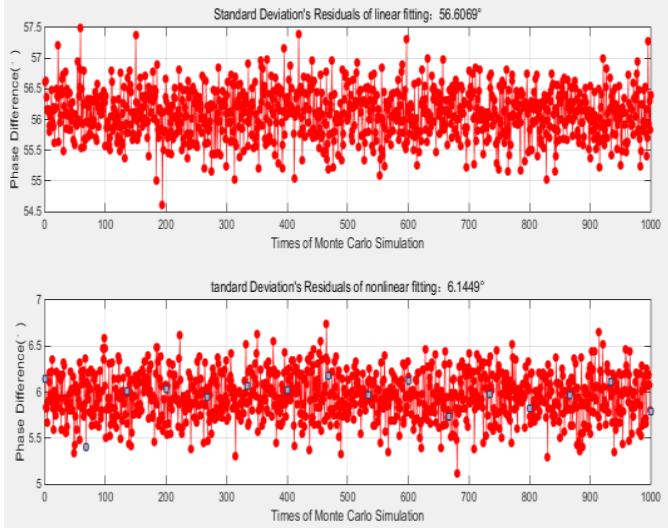

Fig.4 Figure for residuals' standard deviation in method as linear fitting and nonlinear fitting

As the Fig. 3 shows, great fluctuation appears in residuals of linear fitting method, while residuals of nonlinear fitting method fluctuates around zero and is stable. As the Fig. 4 shows, deviation in nonlinear fitting method is much smaller than in linear fitting method. Therefore, linear fitting method is inefficient for wide-band signal.

With the same parameters, mean and standard deviation of addictive phase of difference method and nonlinear fitting method after 1000 Monte Carlo simulation experiments as following form:

Table. 1 The characteristic form for difference method and nonlinear fitting method

\begin{tabular}{cccc}
$\begin{array}{c}\text { Method } \\
\text { Characteristic }\end{array}$ & Difference & Nonlinear Fitting & Remark \\
\hline Mean $\left(^{\circ}\right)$ & 89.7049 & 90.0012 & Addictive Phase $\theta=90^{\circ}$ \\
Standard Deviation $\left(^{\circ}\right)$ & 6.1453 & 0.4824 &
\end{tabular}

As the Table. 1 shows, accuracy of nonlinear fitting method is one more level better than difference method and the former is more accurate.

Thus it is clear that linear fitting method is inefficient for wide-band channel to extract addictive phase and nonlinear fitting method has the best accuracy.

According to definition of group delay and description in chapter 1.2, we can sum up the group delay characteristic curve and the standard deviation curve from the addictive phase characteristic curve. In condition of 1000 Monte Carlo Simulations, there are figures of difference method and nonlinear fitting method as following. Fig. 5 and Fig. 7 respectively shows mean and standard deviation of group delay in difference method, and Fig. 6 and Fig. 8 respectively shows mean and standard deviation of group delay in nonlinear fitting metho

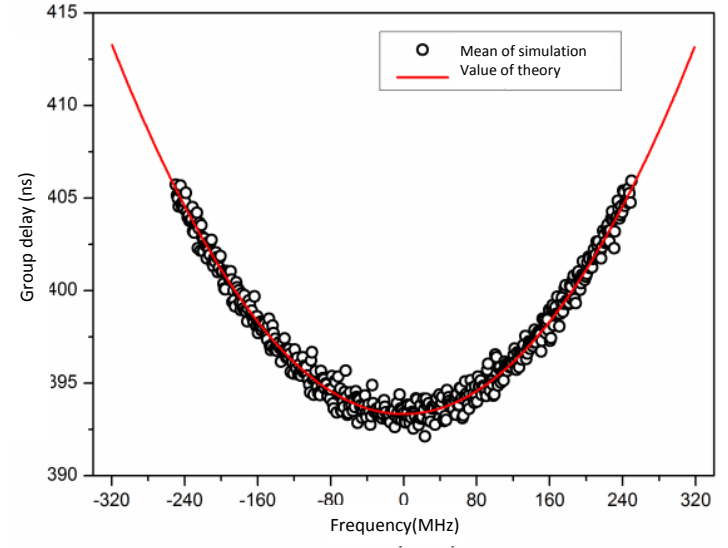

Fig. 5 Figure for means of group delay in difference method

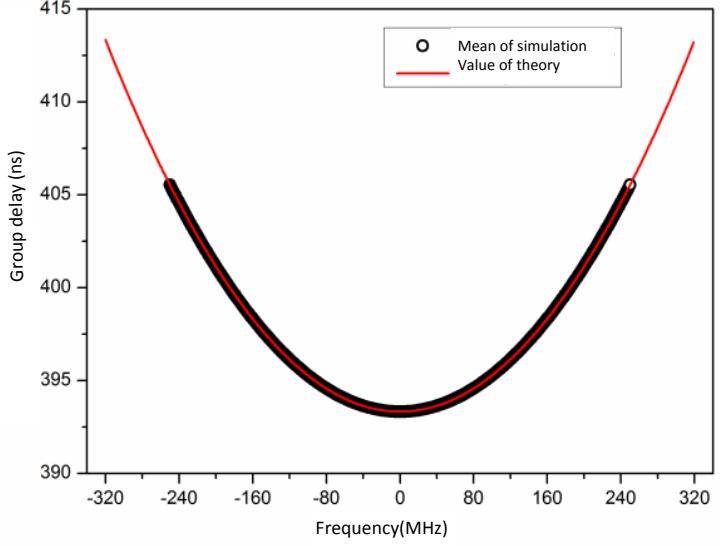

Fig.6 Figure for means of group delay in nonlinear fitting method 


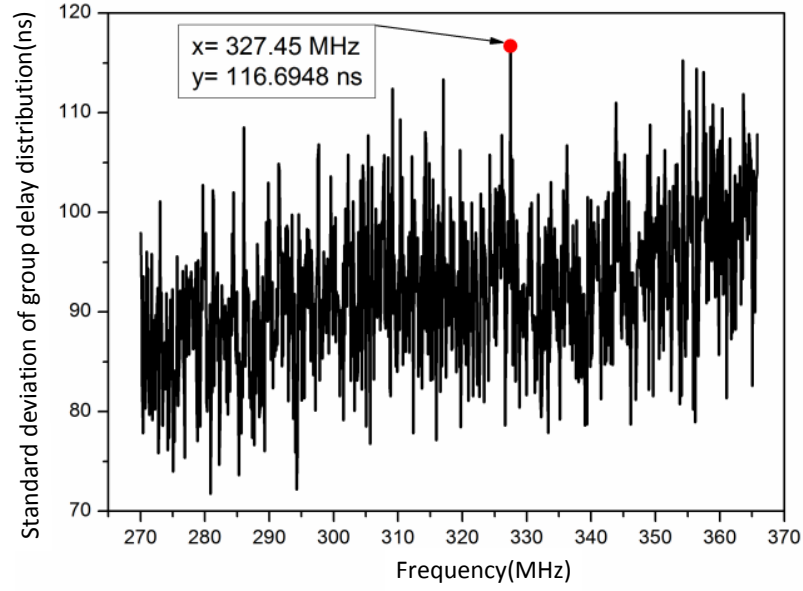

Fig. 7 Figure for standard deviations of group delay in difference method

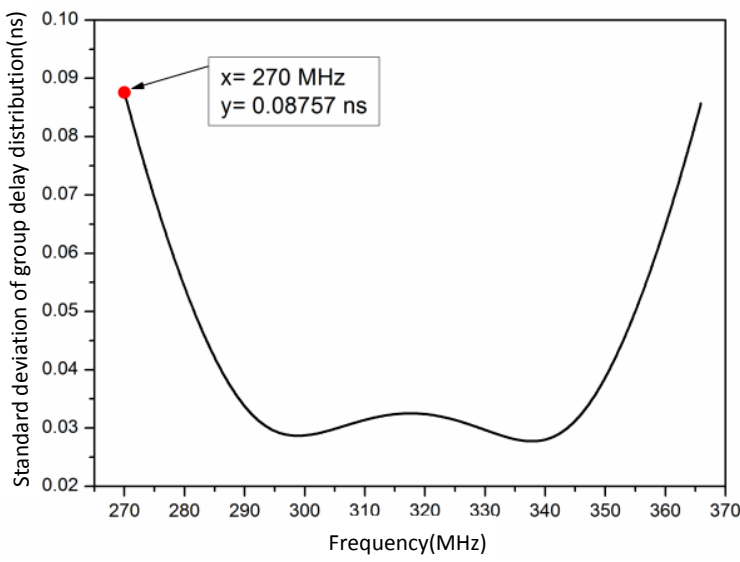

Fig. 8 Figure for standard deviations of group delay in nonlinear fitting method

Sum up above simulations, linear fitting method is inefficient for wide-band channels (nonlinear phase system). Accuracy of difference method is difficult to reach demands of low signal to noise ratio. Nonlinear fitting method effectively solves problems caused by low signal-to-noise ratio and wide bandwidth so as to measure addictive phase and group delay, and the accuracy is improved respectively one order of magnitude of addictive phase and two orders of magnitude of group delay than difference method.

\section{Verification by Measured Data}

July 2016, addictive phase and group delay characteristics of signal of receiving channels in a deep space station are tested with PCAL signals. And then it will be analyzed and studied with measured data as following. The Fig. 9 is a test block figure.

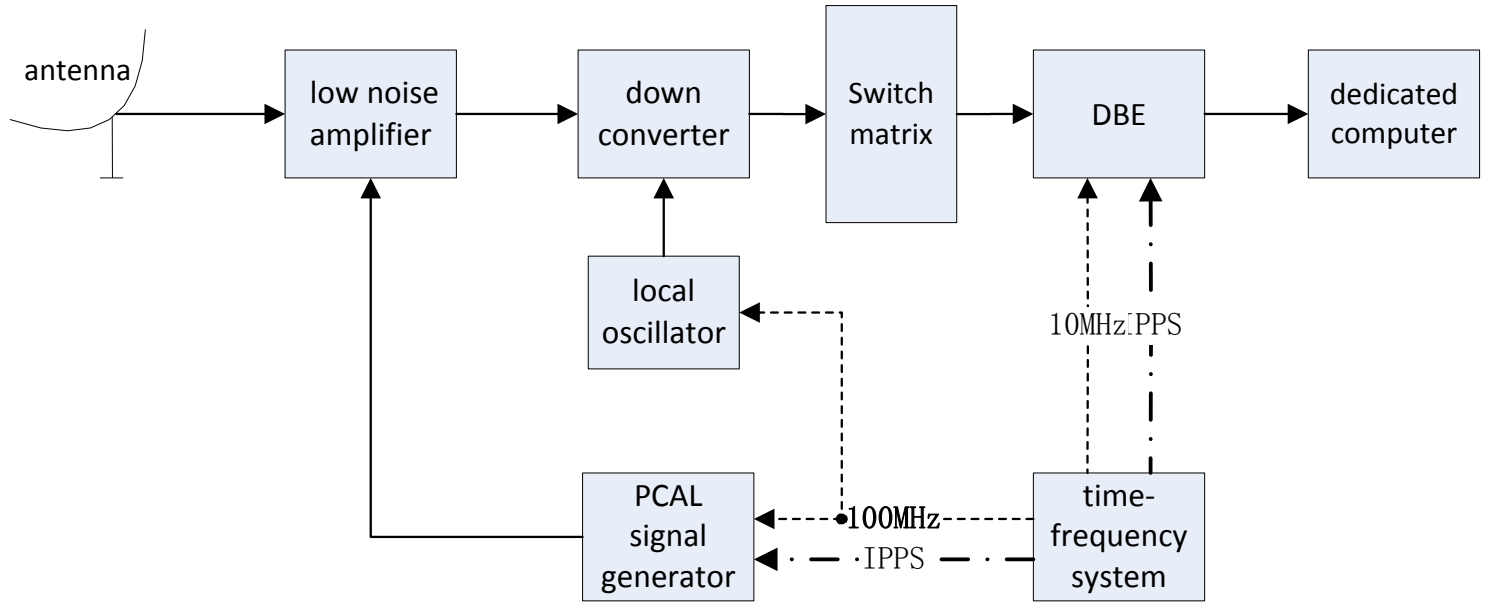

Fig. 9 Figure for test block on PCAL signal

According to the Fig. 9, there are several components of the receiving channels in the Station including low noise amplifier, down converter, filter bank, switch matrix and DBE (Digital baseband converter). Meanwhile, its external systems consist of local oscillator, PCAL signal generator and time-frequency system among these, and time-frequency system is composed of local atomic clock and GPS common view system, which can distribute 10/100MHz signals and IPPS to multi-channels. PCAL signal generators developed by some research institute provide PCAL signals with adjustable power and frequency interval. The basic principle of measurement is that antenna noise and X-band PCAL signals are injected simultaneously into low noise amplifier at the back end of the antenna, down-conversion in down converter, branch signals are transmitted to DBE equipment through switch matrix and finally signals will be correlated in a dedicated computer. During the whole process, time-frequency system provides $100 \mathrm{MHz}$ time-frequency signals for local oscillator and PCAL signal generators, and provides $10 \mathrm{MHz}$ time-frequency signals for DBE. It also provides IPPS signals for 
PCAL signal generators and DBE. The bandwidth of the whole the test channel is $100 \mathrm{MHz}$, from $270 \mathrm{MHz}$ to $370 \mathrm{MHz}$. The main purpose of data-processing is to get phase-frequency characteristic curve and group delay characteristics curve of the channel.

First of all, phase fitting curve and relevant phase residual deviation (fitting deviation) will be got from processing recorded data in linear fitting method and nonlinear fitting method. The result is shown in Fig. 10 and Fig. 11.

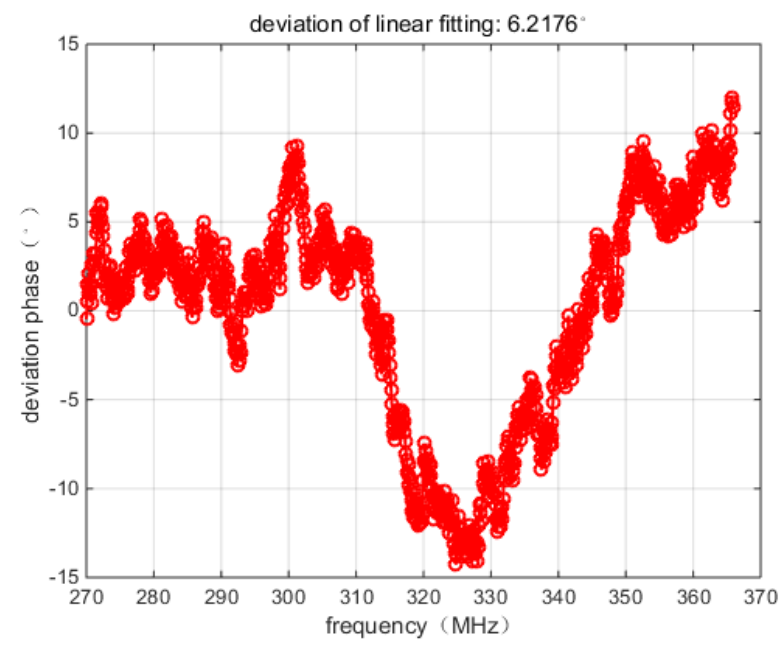

Fig. 10 Figure for deviation of linear fitting method

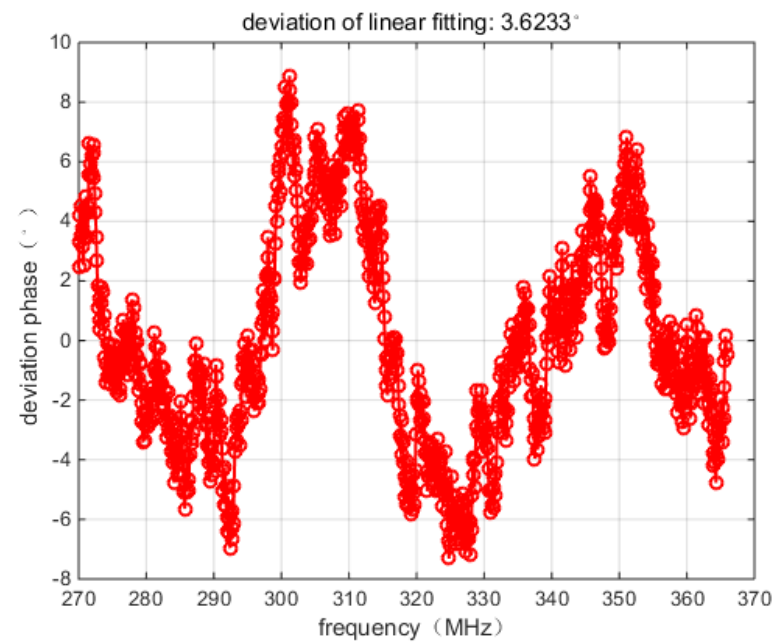

Fig. 11 Figure for deviation of nonlinear fitting method

As the Fig. 10 and Fig. 11 shows, fitting residual deviations of linear fitting method and nonlinear fitting method are respectively $6.2176^{\circ}$ and $3.6233^{\circ}$. The result of nonlinear fitting method is better than linear fitting method. Meanwhile, in residual plot with linear fitting method, mean obviously deviates from zero (non-zero distribution), which gives a reflection that wide-band receiving channels belong to nonlinear phase system. Thus from the measured data above, it can be shown that results of nonlinear fitting method are better than linear fitting method.

Measured data are processed for group delay characteristics of receiving channels using difference method and nonlinear fitting method respectively. Fig. 12 and Fig. 13 respectively shows the results of two methods (difference method and nonlinear fitting method) and includes mean of group delay and characteristics of standard deviation. In the mean figures in Fig. 12 and Fig. 13, results of difference method fluctuate up and down too much, which is hard to demonstrate group delay characteristics of receiving channels. Conversely, results of nonlinear fitting method has a small adulation and is relatively smooth, which demonstrates group delay characteristics of receiving channels effectively. Meanwhile, as the standard deviation figure in Fig. 13 shown, its fluctuation maintains within 1ns, which meets group delay precision of receiving channels and baseband converter [12]. Data have lost their reference value because standard deviation of difference method floats around 700ns. Thus it can be seen that wide-band system measured by nonlinear fitting system can measure addictive phase and group delay characteristics with high accuracy. 


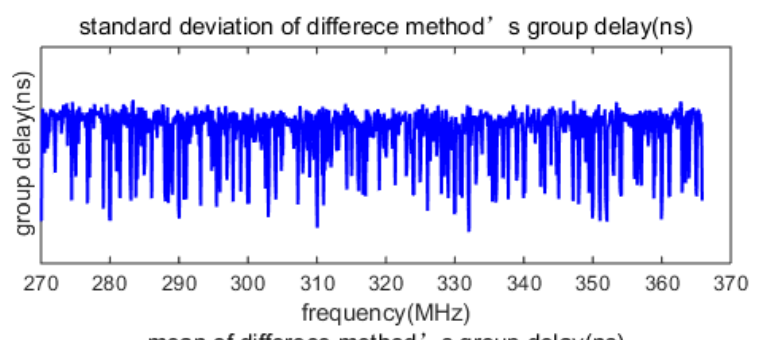

mean of differece method' s group delay(ns)

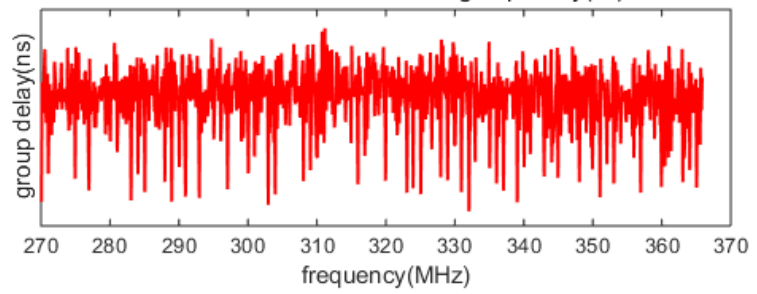

Fig. 12 Figure for mean and Standard deviation of group delay in difference method
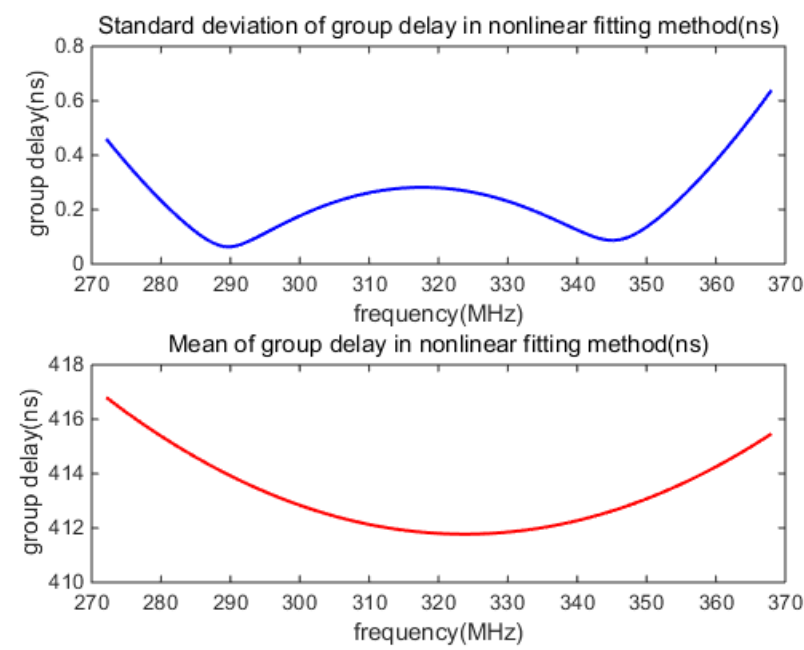

Fig. 13 Figure for mean and Standard deviation of group delay in nonlinear fitting method

\section{Conclusion}

For two issues, one is that difference method is hard to resolve conflicts between resolution of the device and measuring accuracy. The other is that linear fitting method cannot effectively fit the measurement for wide-band channels. In the thesis, the method, measuring group delay characteristics with nonlinear fitting method, is proposed to deal with issues discussed above. On top of introducing three methods, it also proves that only nonlinear fitting method can effectively measure group delay for wide-band channels by means of simulating experiments. At last, superiority of nonlinear fitting method is validated with estimation of group delay characteristics and actual data of PCAL signals in the Deep Space Station. Actual data estimate relatively group delay characteristic curve of bandwidth from $270 \mathrm{MHz}$ to $370 \mathrm{MHz}$ in the Station receive channels and provide a reference for subsequent measuring methods.

\section{References}

[1] Hao Wanhong, Dong Guangliang, Li Haitao. An Introduction to Application of Radio Interferometry in Deep Space Navigation [J]. Journal of Spacecraft TT \& C Technology. 2009, 28(4): $1-7$.

[2] Liu Qinghui, Shi Xian, Wang Weihua etc. High-Accuracy determination of very long baseline interferometry phase delays and its applications in China's future missions [J]. Physics, 2009, 38(10):712-716.

[3] Qian Zhihan, Li Jinling. Application of very long baseline interferometry in deep space [M]. Beijing: China Science and Technology Press, 2012: 2-3.

[4] Li Yuanfei, Zheng Weimin. Research on processing method of VLBI data software [J]. Annals of Shanghai Astronomical Observatory, Chinese Academy of Sciences. 2004(25): 150-158.

[5] Tan Zhugen, Zhai Ning, Chen Yongqiang. The Principle and Implementation of PCAL Signal [J]. Radio Engineering, 2015(6): 32-34.

[6] Jiang Kun, Hou Xiaomin, Xu Ke, etc. High Efficiency Parallel of Multi-Tone PCAL Signals [J]. Journal of Spacecraft TT \& C Technology. 2012, 31(06):32-36.

[7] Jiang Kun, Wang Yuanqin, Hou Xiaomin, etc. High Efficient Extraction of Phase Calibration Signals and Error Analysis [J]. Journal of Signal Processing. 2014, 30(2): 197-204. 
[8] Navarro R, Bunton J. Signal Processing in the Deep Space Array Network [R]. California: JPL IPN Progress Report 42-157, 2004.

[9] Xiao Jianzhong, Zhu Xinghua. Examples on the function and power series expansion coefficient equal to the method of proof [J]. Studies College Mathematics, 2009, 12(3):4-7.

[10] Jovanovic-Dolecek G， Diaz-Carmona J. Digital all-pass filter design method based on complex cepstrum [J]. Electronics Letters. 2003, 39(8): 695-697.

[11] Wang Mei, Tang Geshi, Chen Lue, etc. Interferometric wideband signal phase ambiguity problem [C] China Satellite Navigation Conference. 2012.

[12] Whitney Alan R. VLBI Digital-Backend Inter comparion Testing (Draft) [DB/OL]. http://www.haystack. mit.edu/geo/vlbi_td/BBDev/036.pdf, 2009-5-25. 